\title{
Information-Theoretic Bounds on Quantum Advantage in Machine Learning
}

\author{
Hsin-Yuan Huang $\oplus^{1,2}$ Richard Kueng, ${ }^{3}$ and John Preskill $\oplus^{1,2,4,5}$ \\ ${ }^{1}$ Institute for Quantum Information and Matter, Caltech, Pasadena, California 91125, USA \\ ${ }^{2}$ Department of Computing and Mathematical Sciences, Caltech, Pasadena, California 91125, USA \\ ${ }^{3}$ Institute for Integrated Circuits, Johannes Kepler University Linz, Linz, 4040, Austria \\ ${ }^{4}$ Walter Burke Institute for Theoretical Physics, Caltech, Pasadena, California 91125, USA \\ ${ }^{5}$ AWS Center for Quantum Computing, Pasadena, California 91125, USA
}

(Received 12 January 2021; revised 17 March 2021; accepted 2 April 2021; published 14 May 2021)

\begin{abstract}
We study the performance of classical and quantum machine learning (ML) models in predicting outcomes of physical experiments. The experiments depend on an input parameter $x$ and involve execution of a (possibly unknown) quantum process $\mathcal{E}$. Our figure of merit is the number of runs of $\mathcal{E}$ required to achieve a desired prediction performance. We consider classical ML models that perform a measurement and record the classical outcome after each run of $\mathcal{E}$, and quantum ML models that can access $\mathcal{E}$ coherently to acquire quantum data; the classical or quantum data are then used to predict the outcomes of future experiments. We prove that for any input distribution $\mathcal{D}(x)$, a classical ML model can provide accurate predictions on average by accessing $\mathcal{E}$ a number of times comparable to the optimal quantum ML model. In contrast, for achieving an accurate prediction on all inputs, we prove that the exponential quantum advantage is possible. For example, to predict the expectations of all Pauli observables in an $n$-qubit system $\rho$, classical ML models require $2^{\Omega(n)}$ copies of $\rho$, but we present a quantum ML model using only $\mathcal{O}(n)$ copies. Our results clarify where the quantum advantage is possible and highlight the potential for classical ML models to address challenging quantum problems in physics and chemistry.
\end{abstract}

DOI: 10.1103/PhysRevLett.126.190505

Introduction.-The widespread applications of machine learning (ML) to practical problems have fueled interest in machine learning using quantum platforms [1-3]. Though many potential applications of quantum ML have been proposed, so far the prospect for a quantum advantage in solving purely classical problems remains unclear [4-7]. On the other hand, it seems plausible that quantum ML can be fruitfully applied to problems faced by quantum scientists, such as characterizing the properties of quantum systems and predicting the outcomes of quantum experiments [8-14].

Here, we focus on an important class of learning problems motivated by quantum mechanics. Namely, we are interested in predicting functions of the form

$$
f(x)=\operatorname{tr}(O \mathcal{E}(|x\rangle\langle x|)),
$$

where $x$ is a classical input, $\mathcal{E}$ is an arbitrary (possibly unknown) completely positive and trace preserving (CPTP) map, and $O$ is a known observable. Equation (1) encompasses any physical process that takes a classical input and produces a real number as output. The goal is to construct a function $h(x)$ that accurately approximates $f(x)$ after accessing the physical process $\mathcal{E}$ as few times as possible.

A particularly important special case of setup (1) is training a ML model to predict what would happen in physical experiments [12]. Such experiments might explore, for instance, the outcome of a reaction in quantum chemistry [15], ground-state properties of a novel molecule or material [11,16-21], or the behavior of neutral atoms in an analog quantum simulator [22-24]. In these cases, the input $x$ subsumes parameters that characterize the process, e.g., chemicals involved in the reaction, a description of the molecule, or the intensity of lasers that control the neutral atoms. The map $\mathcal{E}$ characterizes a quantum evolution happening in the lab. Depending on the parameter $x$, it produces the quantum state $\mathcal{E}(|x\rangle\langle x|)$. Finally, the experimentalist measures a certain observable $O$ at the end of the experiment. The goal is to predict the measurement outcome for new physical experiments, with new values of $x$ that have not been encountered during the training process.

Motivated by these concrete applications, we want to understand the power of classical and quantum ML models in predicting functions of the form given in Eq. (1). On the one hand, we consider classical ML models that can gather classical measurement data $\left\{\left(x_{i}, o_{i}\right)\right\}_{i=1}^{N_{\mathrm{C}}}$, where $o_{i}$ is the outcome when we perform a POVM measurement on the state $\mathcal{E}\left(\left|x_{i}\right\rangle\left\langle x_{i}\right|\right)$. We denote by $N_{\mathrm{C}}$ the number of such experiments performed during training in the classical ML setting. On the other hand, we consider quantum ML models in which multiple runs of the CPTP map $\mathcal{E}$ can be composed coherently to collect quantum data, and predictions are produced by a quantum computer with access to the quantum data. We denote by $N_{\mathrm{Q}}$ the number of times 

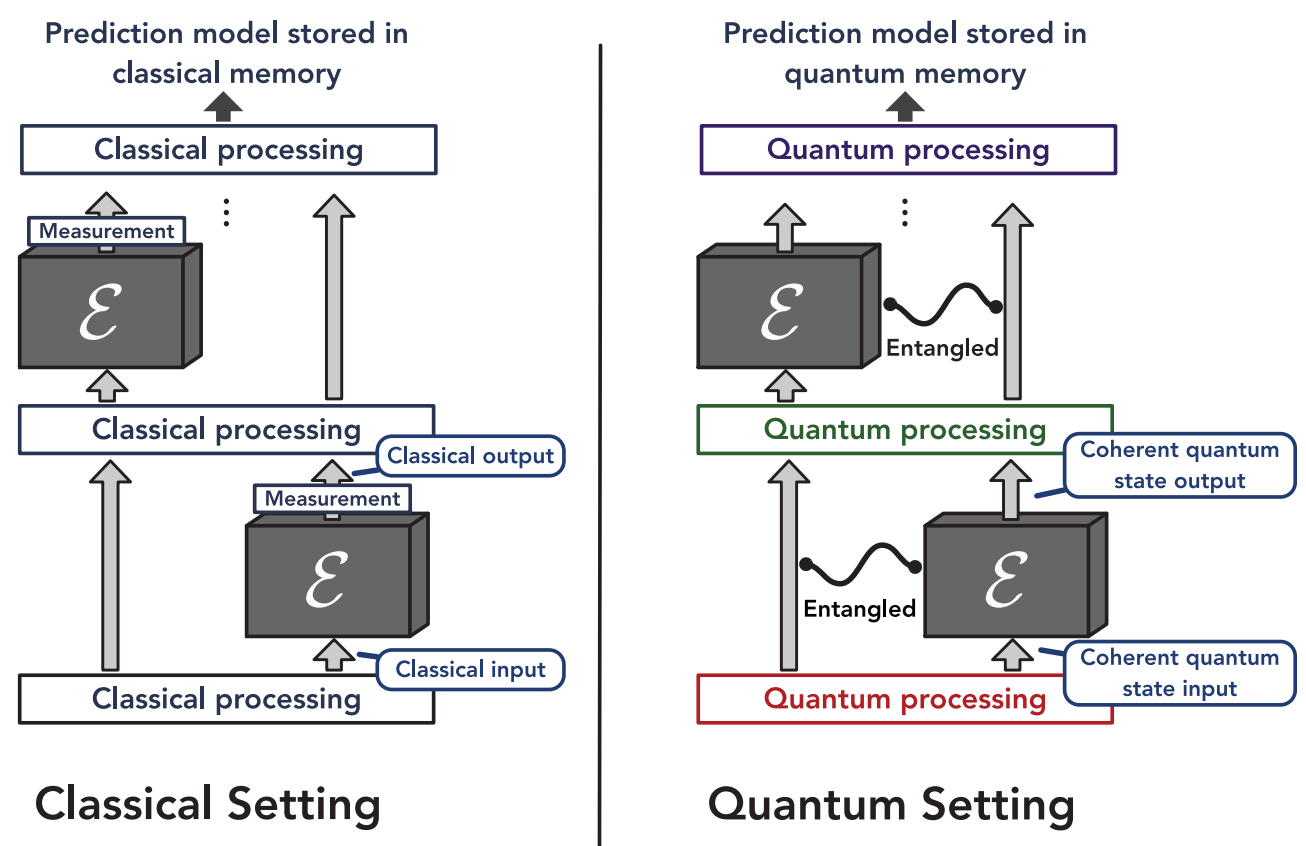

\section{Quantum Setting}

FIG. 1. Illustration of classical and quantum machine learning settings. The goal is to learn about an unknown CPTP map $\mathcal{E}$ by performing physical experiments. Left panel: in the learning phase of the classical ML setting, a measurement is performed after each query to $\mathcal{E}$; the classical measurement outcomes collected during the learning phase are consulted during the prediction phase. Right panel: in the learning phase of the quantum ML setting, multiple queries to $\mathcal{E}$ may be included in a single coherent quantum circuit, yielding an output state stored in a quantum memory; this stored quantum state is consulted during the prediction phase.

$\mathcal{E}$ is used during training in the quantum setting. The classical and quantum ML settings are illustrated in Fig. 1.

We focus on the question of whether quantum ML can have a large advantage over classical ML: To achieve a small prediction error, can the optimal $N_{\mathrm{Q}}$ in the quantum ML setting be much less than the optimal $N_{\mathrm{C}}$ in the classical ML setting? For the purpose of this comparison, we disregard the runtime of the classical or quantum ML models that generate the predictions; we are only interested in how many times the process $\mathcal{E}$ must run during the learning phase in the quantum and classical settings.

Our first main result addresses a small average prediction error, i.e., the prediction error $|h(x)-f(x)|^{2}$ averaged over some specified input distribution $\mathcal{D}(x)$. We rigorously show that, for any $\mathcal{E}, O$, and $\mathcal{D}$, and for any quantum ML model, one can always design a classical ML model achieving a similar average prediction error such that $N_{\mathrm{C}}$ is larger than $N_{\mathrm{Q}}$ by at worst a small polynomial factor. Hence, there is no exponential advantage of quantum ML over classical ML if the goal is to achieve a small average prediction error and if the efficiency is quantified by the number of times $\mathcal{E}$ is used in the learning process. This statement holds for existing quantum ML models running on near-term devices $[2,3,25]$ and future quantum $\mathrm{ML}$ models yet to be conceived. We note, though, that while there is no large advantage in query complexity, a substantial quantum advantage in computational complexity is possible [26].
However, the situation changes if the goal is to achieve a small worst-case prediction error rather than a small average prediction error - an exponential separation between $N_{\mathrm{C}}$ and $N_{\mathrm{Q}}$ becomes possible if we insist on predicting $f(x)=$ $\operatorname{tr}(O \mathcal{E}(|x\rangle\langle x|))$ accurately for every input $x$. We illustrate this point with an example: accurately predicting expectation values of Pauli observables in an unknown $n$-qubit quantum state $\rho$. This is a crucial subroutine in many quantum computing applications; see, e.g., Refs. [20,27-33]. We present a quantum ML model that uses $N_{\mathrm{Q}}=\mathcal{O}(n)$ copies of $\rho$ to predict expectation values of all $n$-qubit Pauli observables. In contrast, we prove that any classical ML model requires $N_{\mathrm{C}}=2^{\Omega(n)}$ copies of $\rho$ to achieve the same task even if the ML model can perform arbitrary, adaptive, single-copy POVM measurements.

Machine learning settings.-We assume that the observable $O$ (with $\|O\| \leq 1$ ) is known, and the physical experiment $\mathcal{E}$ is an unknown CPTP map that belongs to a set of CPTP maps $\mathcal{F}$. Apart from $\mathcal{E} \in \mathcal{F}$, the process can be arbitrary - a common assumption in statistical learning theory [34-38]. For the sake of concreteness, we assume that $\mathcal{E}$ is a CPTP map from a Hilbert space of $n$ qubits to a Hilbert space of $m$ qubits. Regarding inputs, we consider bit strings of size $n: x \in\{0,1\}^{n}$. This is not a severe restriction since floating-point representations of continuous parameters can always be truncated to a finite number of digits. We now give precise definitions for classical and quantum ML settings; see Fig. 1 for an illustration. 
Classical (C) ML.-The ML model consists of two phases: learning and prediction. During the learning phase, a randomized algorithm selects classical inputs $x_{i}$, and we perform a (quantum) experiment that results in an outcome $o_{i}$ from performing a POVM measurement on $\mathcal{E}\left(\left|x_{i}\right\rangle\left\langle x_{i}\right|\right)$. A total of $N_{\mathrm{C}}$ experiments give rise to the classical training data $\left\{\left(x_{i}, o_{i}\right)\right\}_{i=1}^{N_{\mathrm{C}}}$. After obtaining this training data, the ML model executes a randomized algorithm $\mathcal{A}$ to learn a prediction model

$$
s_{\mathrm{C}}=\mathcal{A}\left(\left\{\left(x_{1}, o_{1}\right), \ldots\left(x_{N_{\mathrm{C}}}, o_{N_{\mathrm{C}}}\right)\right\}\right),
$$

where $s_{\mathrm{C}}$ is stored in the classical memory. In the prediction phase, a sequence of new inputs $\tilde{x}_{1}, \tilde{x}_{2}, \ldots \in\{0,1\}^{n}$ is provided. The ML model uses $s_{\mathrm{C}}$ to evaluate predictions $h_{\mathrm{C}}\left(\tilde{x}_{1}\right), h_{\mathrm{C}}\left(\tilde{x}_{2}\right), \ldots$ that approximate $f\left(\tilde{x}_{1}\right), f\left(\tilde{x}_{2}\right), \ldots$ up to small errors.

Restricted classical ML.-We also consider a restricted version of the classical setting. Rather than performing arbitrary POVM measurements, we restrict the ML model to measure the target observable $O$ on the output state $\mathcal{E}\left|x_{i}\right\rangle\left\langle x_{i}\right|$ to obtain the measurement outcome $o_{i}$. In this case, we always have $o_{i} \in \mathbb{R}$ and $\mathbb{E}\left[o_{i}\right]=\operatorname{tr}\left(O \mathcal{E}\left(\left|x_{i}\right\rangle\left\langle x_{i}\right|\right)\right)$.

Quantum (Q) ML.-During the learning phase, the model starts with an initial state $\rho_{0}$ in a Hilbert space of arbitrarily high dimension. Subsequently, the quantum ML model accesses the unknown CPTP map $\mathcal{E}$ a total of $N_{\mathrm{Q}}$ times. These queries are interleaved with quantum data processing steps:

$$
\rho_{\mathcal{E}}=\mathcal{C}_{N_{\mathrm{Q}}}(\mathcal{E} \otimes \mathcal{I}) \mathcal{C}_{N_{\mathrm{Q}}-1} \ldots \mathcal{C}_{1}(\mathcal{E} \otimes \mathcal{I})\left(\rho_{0}\right),
$$

where each $\mathcal{C}_{i}$ is an arbitrary but known CPTP map, and we write $\mathcal{E} \otimes \mathcal{I}$ to emphasize that $\mathcal{E}$ acts on an $n$-qubit subsystem of a larger quantum system. The final state $\rho_{\mathcal{E}}$, encoding the prediction model learned from the queries to the unknown CPTP map $\mathcal{E}$, is stored in a quantum memory. In the prediction phase, a sequence of new inputs $\tilde{x}_{1}, \tilde{x}_{2}, \ldots \in\{0,1\}^{n}$ is provided. A quantum computer with access to the stored quantum state $\rho_{\mathcal{E}}$ executes a computation to produce prediction values $h_{\mathrm{Q}}\left(\tilde{x}_{1}\right), h_{\mathrm{Q}}\left(\tilde{x}_{2}\right), \ldots$ that approximate $f\left(\tilde{x}_{1}\right), f\left(\tilde{x}_{2}\right), \ldots$ up to small errors. [Because of the noncommutativity of quantum measurements, the ordering of new inputs matters. For instance, the two lists $\tilde{x}_{1}, \tilde{x}_{2}$ and $\tilde{x}_{2}, \tilde{x}_{1}$ can lead to different outcome predictions $h_{\mathrm{Q}}\left(\tilde{x}_{i}\right)$. Our main results do not depend on this subtletythey are valid, irrespective of the prediction input ordering.]

The quantum ML setting is strictly more powerful than the classical ML setting. During the prediction phase, classical ML models are restricted to processing classical data, albeit data obtained by measuring a quantum system during the learning phase. In contrast, quantum ML models can work directly with the quantum data and perform quantum data processing. A quantum ML model can have an exponential advantage relative to classical ML models for some tasks, as we demonstrate in Sec. IV.

Average-case prediction error.-For a prediction model $h(x)$, we consider the average-case prediction error

$$
\sum_{x \in\{0,1\}^{n}} \mathcal{D}(x)|h(x)-\operatorname{tr}(O \mathcal{E}(|x\rangle\langle x|))|^{2},
$$

with respect to a fixed distribution $\mathcal{D}$ over inputs, which could, for instance, be the uniform distribution.

Although learning from quantum data is strictly more powerful than learning from classical data, there are fundamental limitations. The following rigorous statement limits the potential for a quantum advantage.

Theorem 1: Fix an $n$-bit probability distribution $\mathcal{D}$, an $m$-qubit observable $O(\|O\| \leq 1)$, and a set $\mathcal{F}$ of CPTP maps with $n$ input qubits and $m$ output qubits. Suppose there is a quantum ML model that accesses the map $\mathcal{E} \in \mathcal{F}$ $N_{\mathrm{Q}}$ times, producing, with high probability, a function $h_{\mathrm{Q}}(x)$ that achieves

$$
\sum_{x \in\{0,1\}^{n}} \mathcal{D}(x)\left|h_{\mathrm{Q}}(x)-\operatorname{tr}(O \mathcal{E}(|x\rangle\langle x|))\right|^{2} \leq \epsilon .
$$

Then, there is a ML model in the restricted classical setting that accesses $\mathcal{E} N_{\mathrm{C}}=\mathcal{O}\left(m N_{\mathrm{Q}} / \epsilon\right)$ times and produces, with high probability, a function $h_{C}$ that achieves

$$
\sum_{x \in\{0,1\}^{n}} \mathcal{D}(x)\left|h_{\mathrm{C}}(x)-\operatorname{tr}(O \mathcal{E}(|x\rangle\langle x|))\right|^{2}=\mathcal{O}(\epsilon) .
$$

Proof sketch.-The proof consists of two parts. First, we cover the entire set of CPTP maps $\mathcal{F}$ with a maximal packing net, i.e., the largest subset $\mathcal{S}=\left\{\mathcal{E}_{s}\right\}_{s=1}^{|\mathcal{S}|} \subset \mathcal{F}$ such that the functions $f_{\mathcal{E}_{s}}(x)=\operatorname{tr}\left(O \mathcal{E}_{s}(|x\rangle\langle x|)\right)$ obey $\sum_{x \in\{0,1\}^{n}} \mathcal{D}(x)\left|f_{\mathcal{E}_{s}}(x)-f_{\mathcal{E}_{s^{\prime}}}(x)\right|^{2}>4 \epsilon$ whenever $s \neq s^{\prime}$. We then set up a communication protocol as follows. Alice chooses an element $s$ of the packing net uniformly at random, records her choice $s$, and then applies $\mathcal{E}_{s} N_{Q}$ times to prepare a quantum state $\rho_{\mathcal{E}_{s}}$ as in Eq. (3). Alice's random ensemble of quantum states is thus given by

$$
\rho_{\mathcal{E}_{s}} \text { with probability } p_{s}=\frac{1}{|\mathcal{S}|}
$$

for $s=1, \ldots,|\mathcal{S}|$. Alice then sends the randomly sampled quantum state $\rho_{\mathcal{E}_{s}}$ to Bob, hoping that Bob can decode the state $\rho_{\mathcal{E}_{s}}$ to recover her chosen message $s$. Using the quantum ML model, Bob can produce the function $h_{Q, s}(x)$. Because, by assumption, the function $h_{Q, s}(x)$ achieves a small average-case prediction error with high probability, and because the packing net has been constructed so that the functions $\left\{f_{\mathcal{E}_{s}}\right\}$ are sufficiently distinguishable, Bob can determine $s$ successfully with high 
probability. Because Alice chose from among $|\mathcal{S}|$ possible messages, the mutual information of the chosen message $s$ and Bob's measurement outcome must be at least of order $\log |\mathcal{S}|$ bits. According to Holevo's theorem, the Holevo $\chi$ quantity of Alice's ensemble, Eq. (7), upper bounds this mutual information and therefore must also be $\chi=\Omega(\log \mid \mathcal{S}) \mid$. Furthermore, we can analyze how $\chi$ depends on $N_{Q}$, finding that each additional application of $\mathcal{E}_{s}$ can increase $\chi$ by at most $\mathcal{O}(m)$. We conclude that $\chi=\mathcal{O}\left(m N_{\mathrm{Q}}\right)$, yielding the lower bound $N_{\mathrm{Q}}=$ $\Omega(\log (|\mathcal{S}|) / m)$. The lower bound applies to any quantum ML model, where the size $|\mathcal{S}|$ of the packing net depends on the average-case prediction error $\epsilon$. This completes the first part of the proof.

In the second part, we explicitly construct a ML model in the restricted classical setting that achieves a small averagecase prediction error using a modest number of experiments. In this ML model, an input $x_{i}$ is selected by sampling from the probability distribution $\mathcal{D}$, and an experiment is performed in which the observable $O$ is measured in the output quantum state $\mathcal{E}\left(\left|x_{i}\right\rangle\left\langle x_{i}\right|\right)$, obtaining the measurement outcome $o_{i}$, which has the expectation value $\operatorname{tr}\left(O \mathcal{E}\left(\left|x_{i}\right\rangle\left\langle x_{i}\right|\right)\right)$. A total of $N_{\mathrm{C}}$ such experiments are conducted. Then, the ML model minimizes the leastsquares error to find the best fit within the aforementioned maximal packing net $\mathcal{S}$ :

$$
h_{\mathrm{C}}=\operatorname{argmin}_{f \in \mathcal{S}} \frac{1}{N_{\mathrm{C}}} \sum_{i=1}^{N_{\mathrm{C}}}\left|f\left(x_{i}\right)-o_{i}\right|^{2} .
$$

Because the measurement outcome $o_{i}$ fluctuates about the expectation value of $O$, it may be impossible to achieve zero training error. Yet, it is still possible for $h_{\mathrm{C}}$ to achieve a small average-case prediction error, potentially even smaller than the training error. We use properties of maximal packing nets and of quantum fluctuations of measurement outcomes to perform a tight statistical analysis of the average-case prediction error, finding that, with high probability, $\sum_{x \in\{0,1\}^{n}} \mathcal{D}(x)\left|h_{\mathrm{C}}(x)-\operatorname{tr}(O \mathcal{E}(|x\rangle\langle x|))\right|^{2}=\mathcal{O}(\epsilon)$, provided that $N_{\mathrm{C}}$ is of order $\log (|\mathcal{S}|) / \epsilon$.

Finally, we combine the two parts to conclude $N_{\mathrm{C}}=\mathcal{O}\left(m N_{\mathrm{Q}} / \epsilon\right)$. The full proof is in the Supplemental Material, App. C [39].

Theorem 1 shows that all problems that are approximately learnable by a quantum ML model are also approximately learnable by some restricted classical ML model that executes the quantum process $\mathcal{E}$ a comparable number of times. This applies, in particular, to predicting outputs of quantum-mechanical processes. The relation $N_{\mathrm{C}}=\mathcal{O}\left(m N_{\mathrm{Q}} / \epsilon\right)$ is tight. We give an example in the Supplemental Material, App. D [39] with $N_{\mathrm{C}}=\Omega\left(m N_{\mathrm{Q}} / \epsilon\right)$.

For the task of learning classical Boolean circuits, fundamental limits on the quantum advantage have been established in previous work [26,34,40-43]. Theorem 1 generalizes these existing results to the task of learning outcomes of quantum processes.

Worst-case prediction error.-Rather than achieving a small average prediction error, one may be interested in obtaining a prediction model that is accurate for all inputs $x \in\{0,1\}^{n}$. For a prediction model $h(x)$, we consider the worst-case prediction error to be

$$
\max _{x \in\{0,1\}^{n}}|h(x)-\operatorname{tr}(O \mathcal{E}(|x\rangle\langle x|))|^{2} .
$$

Under such a stricter performance requirement, an exponential quantum advantage becomes possible.

We highlight this potential by means of an illustrative and practically relevant example: predicting expectation values of Pauli operators in an unknown $n$-qubit quantum state $\rho$. This is a central task for many quantum computing applications [20,27-33]. To formulate this problem in our framework, suppose the $2 n$-bit input $x$ specifies one of the $4^{n} n$-qubit Pauli operators $P_{x} \in\{I, X, Y, Z\}^{\otimes n}$, and suppose that $\mathcal{E}_{\rho}(|x\rangle\langle x|)$ prepares the unknown state $\rho$ and maps $P_{x}$ to the fixed observable $O$, which is then measured; hence,

$$
f(x)=\operatorname{tr}\left(O \mathcal{E}_{\rho}(|x\rangle\langle x|)\right)=\operatorname{tr}\left(P_{x} \rho\right) .
$$

In this setting, according to Theorem 1, there is no large quantum advantage if our goal is to estimate the Pauli operator expectation values with a small average prediction error. However, an exponential quantum advantage is possible if we insist on accurately predicting every one of the $4^{n}$ Pauli observables.

First, we show that there is an efficient quantum ML model that achieves a small prediction error. Details are given in the Supplemental Material, App. E 2 [39]; here, we just sketch the main ideas. The procedure for predicting $\operatorname{tr}\left(P_{x} \rho\right)$ has two stages. The goal of the first stage is to predict the absolute value $\left|\operatorname{tr}\left(P_{x} \rho\right)\right|$ for each $x$, and the goal of the second stage is to determine the sign of $\operatorname{tr}\left(P_{x} \rho\right)$. The key idea used in the first stage is that, although two different Pauli operators $P_{x}$ and $P_{y}$ may either commute or anticommute, the tensor products $P_{x} \otimes P_{x}$ and $P_{y} \otimes P_{y}$ mutually commute for all $x$ and $y$. Therefore, although it is not possible to measure anticommuting Pauli operators simultaneously using a single copy of the state $\rho$, it is possible to measure $P_{x} \otimes P_{x}$ simultaneously for all $x$ using two copies of $\rho$. Indeed, all $4^{n}$ expectation values $\operatorname{tr}\left(\left(P_{x} \otimes\right.\right.$ $\left.\left.P_{x}\right)(\rho \otimes \rho)\right)=\operatorname{tr}\left(P_{x} \rho\right)^{2}$ can be determined by measuring pairs of qubits in the Bell basis, which is highly efficient. This completes the first stage.

If $\left|\operatorname{tr}\left(P_{x} \rho\right)\right|$ is found to be small in the first stage, we may predict $h(x)=0$ and be assured that the prediction error is small. Therefore, in the second stage, we need only determine the sign if $\left|\operatorname{tr}\left(P_{x} \rho\right)\right|$ is found to be reasonably large in the first stage. In that case, we can 
perform a coherent measurement across several copies of $\rho$, which performs a majority vote and yields the correct value of the sign with a high probability of success. Because the measurement is strongly biased in favor of one of the two possible outcomes, it introduces only a very "gentle" disturbance of the premeasurement state. Therefore, by performing many such measurements in succession on the same quantum memory register, we can determine the sign of $\operatorname{tr}\left(P_{x} \rho\right)$ for many different values of $x$. The second stage can also be more amenable to nearterm implementation using a heuristic that groups commuting observables [30,31]; See the Supplemental Material, App. E 2 d [39] for further discussion. Each of the two stages requires only a small number of copies of $\rho$; a careful analysis yields the following theorem.

Theorem 2: The quantum ML model only needs $N_{\mathrm{Q}}=$ $\mathcal{O}\left(\log (M / \delta) / \epsilon^{4}\right)$ copies of $\rho$ to predict expectation values of any $M$ Pauli observables to an error $\epsilon$ with a probability of at least $1-\delta$.

More details regarding the quantum ML model, as well as a rigorous proof, are provided in the Supplemental Material, App. E 2 [39]. The sample complexity stated in Theorem 2 improves upon previously known shadow tomography protocols $[29,44-46]$ for the special case of predicting Pauli observables; see Supplemental Material, App. A [39]. Because each access to $\mathcal{E}_{\rho}$ allows us to obtain one copy of $\rho$, we only need $N_{\mathrm{Q}}=\mathcal{O}(n)$ to predict expectation values of all $4^{n}$ Pauli observables up to a constant error.

For classical ML models, we prove the following fundamental lower bound; see Supplemental Material, App. E 4 [39].

Theorem 3: Any classical ML must use $N_{\mathrm{C}} \geq 2^{\Omega(n)}$ copies of $\rho$ to predict expectation values of all Pauli observables up to a small error with a constant success probability.

This theorem holds even when the POVM measurements performed by the classical ML model depend on the previous POVM measurement outcomes adaptively. When combined with Theorem 2, Theorem 3 establishes an exponential gap separating classical ML models

\begin{tabular}{c|c|c} 
Model & Upper bound & Lower bound \\
\hline Quantum ML & $\mathcal{O}(n)$ & $\Omega(n)$ \\
Classical ML & $\mathcal{O}\left(n 2^{n}\right)$ & $2^{\Omega(n)}$ \\
Restricted CML & $\mathcal{O}\left(2^{2 n}\right)$ & $\Omega\left(2^{2 n}\right)$
\end{tabular}

FIG. 2. Sample complexity for predicting expectations of all $4^{n}$ Pauli observables (worst-case prediction error) in an $n$-qubit quantum state. "Upper bound" is the achievable sample complexity of a specific algorithm. "Lower bound" is the lower bound for any algorithm. The classical ML upper bound can be achieved using classical shadows based on random Clifford measurements [29]. The rest of the bounds are obtained in the Supplemental Material, App. E [39]. from fully quantum ML models. Figure 2 provides a summary of the upper and lower bounds on the sample complexity for predicting expectation values of Pauli observables.

Numerical experiments. -We support our theoretical findings with numerical experiments, focusing on the task of predicting the expectation values of all $4^{n}$ Pauli observables in an unknown $n$-qubit quantum state $\rho$, with a small worst-case prediction error. In this case, the function is $f(x)=\operatorname{tr}\left(O \mathcal{E}_{\rho}(|x\rangle\langle x|)\right)=\operatorname{tr}\left(P_{x} \rho\right)$, where $x \in$ $\{I, X, Y, Z\}^{n}$ indexes the Pauli observables, and $\mathcal{E}_{\rho}$ prepares the unknown state $\rho$ and then maps $P_{x}$ to the fixed observable $O$. This is the task we considered previously. Note that the average-case prediction of Pauli observables is a much easier task because most of the $4^{n}$ expectation values are exponentially small in $n$.

We consider two classes of underlying states $\rho$ : (i) Mixed states: $\rho=(I+P) / 2^{n}$, where $P$ is a tensor product of $n$ Pauli operators. States in this class have rank $2^{n-1}$. (ii) Product states: $\rho=\bigotimes_{i=1}^{n}\left|s_{i}\right\rangle\left\langle s_{i}\right|$, where each $\left|s_{i}\right\rangle$ is one of the six possible single-qubit stabilizer states. We consider stabilizer states to ensure that classical simulation of the quantum ML model is tractable for a reasonably large system size.

The numerical experiment in Fig. 3 implements the best-known ML procedures. We can clearly see that there is an exponential separation between the number of copies of the state $\rho$ required for classical and quantum ML to predict expectation values when $\rho$ is in the class of mixed states. However, for the class of product states, the separation is much less pronounced. Restricted classical ML can only obtain outcomes $o_{i} \in\{ \pm 1\}$ with $\mathbb{E}\left[o_{i}\right]=\operatorname{tr}\left(P_{x_{i}} \rho\right)$. Hence, each copy of $\rho$ provides at most one bit of information, and therefore $\mathcal{O}(n)$ copies are needed to predict expectation values of all $4^{n}$ Pauli observables. In contrast, standard classical ML can perform arbitrary POVM measurements on the state $\rho$, so each copy can provide up to $n$ bits of information. The
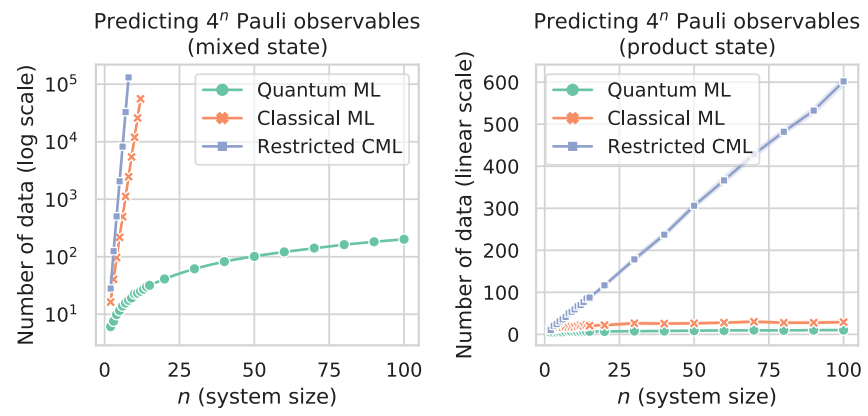

FIG. 3. Numerical experiments-number of copies of an unknown $n$-qubit state needed for predicting expectation values of all $4^{n}$ Pauli observables, with a constant worst-case prediction error. Mixed states: quantum states of the form $(I+P) / 2^{n}$, where $P$ is an $n$-qubit Pauli observable. Product states: tensor products of single-qubit stabilizer states. 
separation between classical ML and quantum ML is marginal for product states.

Conclusion and outlook.-We have studied the task of learning functions of the form in Eq. (1), using as a figure of merit the number of runs of $\mathcal{E}$. Our main result, Theorem 1 , shows that, when the objective is achieving a specified average prediction error, a classical ML model can perform as well as a quantum ML model, using a comparable number of runs of $\mathcal{E}$. This result establishes a fundamental limit on the quantum advantage in machine learning that holds for any quantum ML model [2,3,25].

From a different perspective, Theorem 1 means that the classical ML setting, in which a measurement is performed after each query to $\mathcal{E}$, can be surprisingly effective. The quantum ML setting, in which multiple queries to $\mathcal{E}$ can be included in a single coherent quantum circuit, is far more challenging and may not be feasible until far in the future. Therefore, finding that classical and quantum ML have comparable power (for the average-case prediction) boosts our hopes that the combination of classical ML and nearterm quantum algorithms $[2,3,25,28,47]$ may fruitfully address challenging quantum problems in physics, chemistry, and materials science.

On the other hand, Theorems 2 and 3 rigorously establish that quantum ML can have an exponential advantage over classical ML for certain problems where the objective is to achieve a specified worst-case prediction error. This exponential advantage of quantum ML over classical ML may be viewed as an exponential separation between coherent measurements (in which a measurement apparatus interacts coherently multiple times with a measured system, storing quantum data that are then processed by a quantum computer) and incoherent measurements (in which a POVM measurement is performed and the outcome recorded after each interaction between the system and apparatus, and the classical measurement outcomes are then processed by a classical computer). Such a separation has been challenging to establish because incoherent measurements are difficult to analyze in the adaptive setting, where each measurement performed may depend on the outcomes of all previous measurements. Our proof technique overcomes this challenge, enabling us to identify tasks that allow a substantial quantum advantage. An important future direction will be to identify further learning problems that allow a substantial quantum advantage, pointing toward potential practical applications of quantum technology.

The authors thank Victor Albert, Sitan Chen, Jerry Li, Seth Lloyd, Jarrod McClean, Spiros Michalakis, Yuan Su, and Thomas Vidick for valuable input and inspiring discussions. We would also like to thank anonymous reviewers for in-depth comments and suggestions. H. H. is supported by the J. Yang \& Family Foundation. J. P. acknowledges funding from the U.S. Department of Energy, Office of Science, Office of Advanced Scientific
Computing Research, (Grants No. DE-NA0003525 and No. DE-SC0020290), and the National Science Foundation (Grant No. PHY-1733907). The Institute for Quantum Information and Matter is a NSF Physics Frontiers Center.

[1] J. Biamonte, P. Wittek, N. Pancotti, P. Rebentrost, N. Wiebe, and S. Lloyd, Quantum machine learning, Nature (London) 549, 195 (2017).

[2] V. Havlíček, A. D. Córcoles, K. Temme, A. W. Harrow, A. Kandala, J. M. Chow, and J. M. Gambetta, Supervised learning with quantum-enhanced feature spaces, Nature (London) 567, 209 (2019).

[3] M. Schuld and N. Killoran, Quantum Machine Learning in Feature Hilbert Spaces, Phys. Rev. Lett. 122, 040504 (2019).

[4] J. M. Arrazola, A. Delgado, B. R. Bardhan, and S. Lloyd, Quantum-inspired algorithms in practice, Quantum 4, 307 (2020).

[5] A. Gilyén, S. Lloyd, and E. Tang, Quantum-inspired lowrank stochastic regression with logarithmic dependence on the dimension, arXiv:1811.04909.

[6] E. Tang, Quantum-inspired classical algorithms for principal component analysis and supervised clustering, arXiv:1811.00414.

[7] E. Tang, A quantum-inspired classical algorithm for recommendation systems, in Proceedings of the 51st Annual ACM SIGACT Symposium on Theory of Computing (2019), pp. 217-228.

[8] D. Aharonov, J. Cotler, and X.-L. Qi, Quantum algorithmic measurement, arXiv:2101.04634.

[9] G. Carleo and M. Troyer, Solving the quantum many-body problem with artificial neural networks, Science 355, 602 (2017).

[10] J. Carrasquilla and R. G. Melko, Machine learning phases of matter, Nat. Phys. 13, 431 (2017).

[11] J. Gilmer, S. S. Schoenholz, P. F. Riley, O. Vinyals, and G. E. Dahl, Neural message passing for quantum chemistry, arXiv: 1704.01212.

[12] A. A. Melnikov, H. P. Nautrup, M. Krenn, V. Dunjko, M. Tiersch, A. Zeilinger, and H. J. Briegel, Active learning machine learns to create new quantum experiments, Proc. Natl. Acad. Sci. U.S.A. 115, 1221 (2018).

[13] O. Sharir, Y. Levine, N. Wies, G. Carleo, and A. Shashua, Deep Autoregressive Models for the Efficient Variational Simulation of Many-Body Quantum Systems, Phys. Rev. Lett. 124, 020503 (2020).

[14] E. P. Van Nieuwenburg, Y.-H. Liu, and S. D. Huber, Learning phase transitions by confusion, Nat. Phys. 13, 435 (2017).

[15] Z. Zhou, X. Li, and R. N. Zare, Optimizing chemical reactions with deep reinforcement learning, ACS Cent. Sci. 3, 1337 (2017).

[16] A. D. Becke, A new mixing of Hartree-Fock and local density-functional theories, J. Chem. Phys. 98, 1372 (1993).

[17] R. Car and M. Parrinello, Unified Approach for Molecular Dynamics and Density-Functional Theory, Phys. Rev. Lett. 55, 2471 (1985).

[18] A. Kandala, A. Mezzacapo, K. Temme, M. Takita, M. Brink, J. M. Chow, and J. M. Gambetta, Hardware-efficient 
variational quantum eigensolver for small molecules and quantum magnets, Nature (London) 549, 242 (2017).

[19] R. G. Parr, Density functional theory of atoms and molecules, in Horizons of Quantum Chemistry (Springer, New York, 1980), pp. 5-15.

[20] A. Peruzzo, J. McClean, P. Shadbolt, M.-H. Yung, X.-Q. Zhou, P. J. Love, A. Aspuru-Guzik, and J. L. O'brien, A variational eigenvalue solver on a photonic quantum processor, Nat. Commun. 5, 4213 (2014).

[21] S. R. White, Density-matrix algorithms for quantum renormalization groups, Phys. Rev. B 48, 10345 (1993).

[22] H. Bernien, S. Schwartz, A. Keesling, H. Levine, A. Omran, H. Pichler, S. Choi, A. S. Zibrov, M. Endres, M. Greiner et al., Probing many-body dynamics on a 51-atom quantum simulator, Nature (London) 551, 579 (2017).

[23] I. Buluta and F. Nori, Quantum simulators, Science 326, 108 (2009).

[24] H. Levine, A. Keesling, A. Omran, H. Bernien, S. Schwartz, A.S. Zibrov, M. Endres, M. Greiner, V. Vuletić, and M. D. Lukin, High-Fidelity Control and Entanglement of Rydberg-Atom Qubits, Phys. Rev. Lett. 121, 123603 (2018).

[25] H.-Y. Huang, M. Broughton, M. Mohseni, R. Babbush, S. Boixo, H. Neven, and J. R. McClean, Power of data in quantum machine learning, arXiv:2011.01938.

[26] R. A. Servedio and S. J. Gortler, Equivalences and separations between quantum and classical learnability, SIAM J. Comput. 33, 1067 (2004).

[27] O. Crawford, B. van Straaten, D. Wang, T. Parks, E. Campbell, and S. Brierley, Efficient quantum measurement of Pauli operators in the presence of finite sampling error, Quantum 5, 385 (2021).

[28] H.-Y. Huang, K. Bharti, and P. Rebentrost, Near-term quantum algorithms for linear systems of equations, arXiv:1909.07344.

[29] H.-Y. Huang, R. Kueng, and J. Preskill, Predicting many properties of a quantum system from very few measurements, Nat. Phys. 16, 1050 (2020).

[30] W. J. Huggins, J. McClean, N. Rubin, Z. Jiang, N. Wiebe, K. B. Whaley, and R. Babbush, Efficient and noise resilient measurements for quantum chemistry on near-term quantum computers, npj Quantum Inf. 7, 23 (2021).

[31] A. F. Izmaylov, T.-C. Yen, R. A. Lang, and V. Verteletskyi, Unitary partitioning approach to the measurement problem in the variational quantum eigensolver method, J. Chem. Theory Comput. 16, 190 (2020).
[32] Z. Jiang, A. Kalev, W. Mruczkiewicz, and H. Neven, Optimal fermion-to-qubit mapping via ternary trees with applications to reduced quantum states learning, Quantum 4, 276 (2020).

[33] C. Kokail, C. Maier, R. van Bijnen, T. Brydges, M. K. Joshi, P. Jurcevic, C. A. Muschik, P. Silvi, R. Blatt, C. F. Roos et al., Self-verifying variational quantum simulation of lattice models, Nature (London) 569, 355 (2019).

[34] S. Arunachalam and R. de Wolf, Guest column: A survey of quantum learning theory, ACM SIGACT News 48, 41 (2017).

[35] P. L. Bartlett and S. Mendelson, Rademacher and Gaussian complexities: Risk bounds and structural results, J. Mach. Learn. Res. 3, 463 (2002).

[36] A. Blumer, A. Ehrenfeucht, D. Haussler, and M. K. Warmuth, Learnability and the Vapnik-Chervonenkis dimension, J. ACM 36, 929 (1989).

[37] L. G. Valiant, A theory of the learnable, Commun. ACM 27, 1134 (1984).

[38] V. Vapnik, The Nature of Statistical Learning Theory (Springer Science \& Business Media, New York, 2013).

[39] See Supplemental Material at http://link.aps.org/supplemental/ 10.1103/PhysRevLett.126.190505 for the detailed proofs.

[40] S. Arunachalam and R. de Wolf, Optimal quantum sample complexity of learning algorithms, arXiv:1607.00932.

[41] S. Arunachalam, A. B. Grilo, and H. Yuen, Quantum statistical query learning, arXiv:2002.08240.

[42] K.-M. Chung and H.-H. Lin, Sample efficient algorithms for learning quantum channels in PAC model and the approximate state discrimination problem, arXiv:1810.10938.

[43] C. Zhang, An improved lower bound on query complexity for quantum PAC learning, Inf. Proc. Lett. 111, 40 (2010).

[44] S. Aaronson, Shadow tomography of quantum states, in SIAM Journal on Computing (2019), pp. 325-338.

[45] S. Aaronson and G. N. Rothblum, Gentle measurement of quantum states and differential privacy, in Proceedings of the 51st Annual ACM SIGACT Symposium on Theory of Computing (2019), pp. 322-333.

[46] C. Bădescu and R. O'Donnell, Improved quantum data analysis, arXiv:2011.10908.

[47] J. Preskill, Quantum computing in the NISQ era and beyond, Quantum 2, 79 (2018). 